\title{
Longissimus lumborum muscle metastasis from mammary gland adenocarcinoma in a dog
}

\author{
Jae-Yeon Lee, Beom-Jun Shin, Ji-Young Park, Young-Won Lee, Seong-Jun Park, \\ Myung-Cheol Kim, Seong-Mok Jeong* \\ College of Veterinary Medicine and Research Institute of Veterinary Medicine, \\ Chungnam National University, Daejeon 305-764, Korea \\ (Received: February 13, 2012; Revised: September 11, 2012; Accepted: September 28, 2012)
}

\begin{abstract}
An 8-year-old female, German Shepherd dog was presented with history of dyspnea and seizure. One Year Previously a lumpectomy had been performed for surgical resection of mammary mass. In serum chemistry, severe hypoglycemia and elevation of aspartate aminitransferase and creatine kinase were shown. In thoracic radiography, there were variable sized nodules in the overall lung field. On computed tomography examination, the mass was shown in left longissimus lumborum muscle. Histopathological examination revealed adenocarcinoma derived from the mammary gland. Muscular metastasis of mammary gland tumor is uncommon. This is a rare observation and could easily be overlooked or misinterpreted.
\end{abstract}

Keywords : dog, longissimus lumborum muscle, mammary adenocarcinoma, metastasis

Mammary gland tumor (MGT) is one of the most common tumors that occur in female dogs, and which increased its annual incidence rate from $25 \sim 50 \%[5,10]$. In dogs, the risk of developing MGT increases after 6 years of age [10]. Several breeds have been reported to be moderately predisposed including toy and miniature poodles, several Spaniel breeds, English setters, pointers, Maltese, Yorkshire terriers, dachshunds and German shepherds may be predisposed more specifically to malignant mammary tumors $[3,9,14]$.

The exact incidence of mammary tumors and the benign/ malignant ratio are difficult to determine, but it can be estimated that approximately 49 percent of the surgically removed mammary tumors are malignant [2]. There are fibroadenomas (benign mixed tumor), simple adenomas and benign mesenchymal tumors in non-malignant tumors and solid carcinoma, tubular, papillary adenocarcinoma, anaplastic carcinomas, sarcomas and carcinosarcoma (malignant mixed tumors) in malignant tumors [2]. Malignant tumors are cancer, where the cancer cells can invade and damage tissues and organs near the tumor. Also, cancer cells can break away from a malignant tumors and enter the lymphatic system or the bloodstream. In addition, other general transition regions include the lung, liver, kidney, and more rarely, bone $[7,13]$. Treatment of malignant tumors may include surgical removal, radiation, or chemotherapy. Sometimes, a combination of surgery, chemotherapy, and radiation is used [10].

The purpose of the current study was to describe a case of a rare muscular metastasis of mammary adenocarcinoma in a dog that presented as dyspnoea and seizure.

An 8-year-old female, German Shepherd dog was presented with a history of dyspnea and seizure. One year ago, lumpectomy was performed for surgical resection of a mammary mass in a military veterinary hospital. A CBC revealed leukocytosis (total number of with blood cell, $28.87 \mathrm{~K} / \mu \mathrm{L}$, reference range $6 \sim 17 \mathrm{~K} / \mu \mathrm{L}$ ), and serum biochemical analyses revealed severe hypoglycemia $(57 \mathrm{mg} / \mathrm{dL}, 67 \sim 139 \mathrm{mg} /$ $\mathrm{dL}$ ), high activities of aspartate aminitransferase (AST; $90 \mu$ / $\mathrm{L}, 7 \sim 84 \mu / \mathrm{L})$ and creatine kinase $(\mathrm{CK} ; 848 \mu / \mathrm{L}, 51 \sim 485 \mu$ / L). In thoracic radiography, there were variable sized nodules in the overall lung field (Fig. 1). In addition, a soft tissue opacity mass was revealed, with a level of lumber which was 2 to 4 in abdominal radiography. On cytologic findings of the mass, macrokayosis, high $\mathrm{N}: \mathrm{C}$ ratio and anisokaryosis in nucleus were observed (Fig. 2).

On computed tomography (CT) examination, the mass was shown in the left longissimus lumborum muscle. In addition, multiple nodules were shown in thoracic regions (Fig. 3). For evaluating brain tumor metastasis, magnetic resonance imaging was performed, but there was no evidence of brain metastasis. The dog was euthanazed. During necropsy, variable sized round shaped nodules were observed in lung parenchyma and hemorrhagic exudates were shown in the thoracic cavity. A muscular mass $(10 \times 5 \times 4 \mathrm{~cm})$ was observed as an infiltrated nodule. Microscopically, the mass

*Corresponding author

Tel: +82-42-821-6763, Fax: +82-42-821-6703

E-mail: jsmok@cnu.ac.kr 


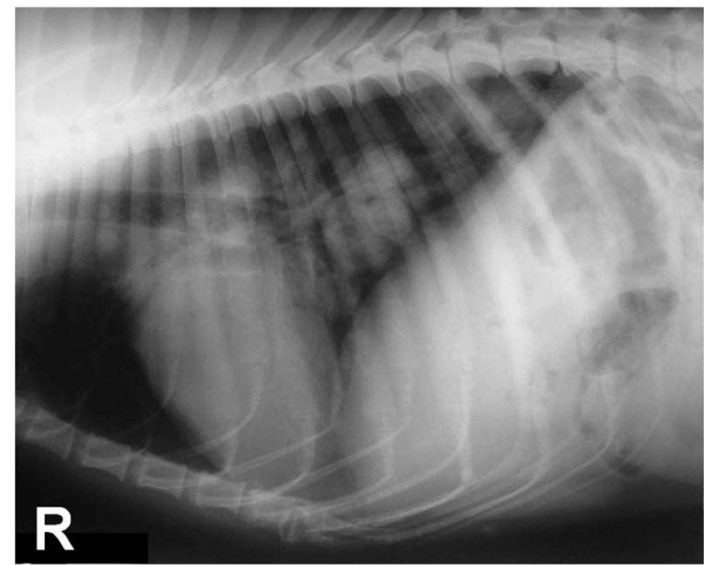

Fig. 1. The lateral view thoracic radiography of a dog. Multiple interstitial nodular pattern is shown in the overall lung field.

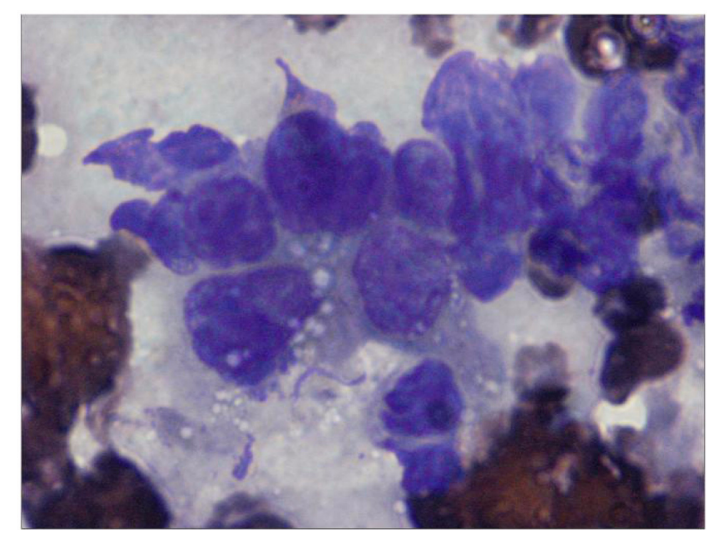

Fig. 2. Fine needle aspirate of the intramuscular mass. Macrokayosis, high $\mathrm{N}: \mathrm{C}$ ratio and anisokaryosis in nucleus is observed. Diff-Quick Stain, $\times 1,000$.

walls were lined by columnar and cubic epithelium (Fig. 4). On the basis of the histopathologic examination, the mass was diagnosed as an adeocarcinoma derived from the mammary gland.

In the case of tumors, systemic complication or abnormal signs can appear regardleess of the tumor size, the presence of metastasis, or physiological activities of tissue of the primary site. These effects are defined as paraneoplastic syndromes [8]. Anemia, thrombocytopenia, hypercalcemia, hypoglycemia, and neurological disorders can occur as paraneoplastic syndromes, and are commonly found in veterinary clinics. Hypoglycemia due to excessive secretion of insulin and excess glucose consumption can appear due to drug-related complications. In this case, although a normal plasma calcium concentration was observed, the level of glucose was extremely low at $57 \mathrm{mg} / \mathrm{dL}$. The predominant sites of MGT invasion were the reproductive system, respiratory system and nervous system. In particular, abdominal and lung metastasis can occur through the inguinal node or internal iliac lymph node [7].

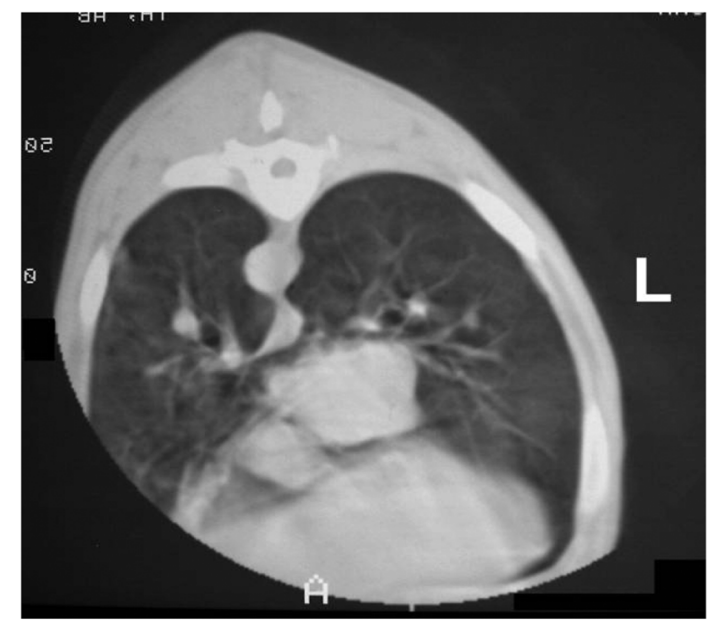

Fig. 3. Multiple nodules of thoracic regions. Computed tomographic examination of a dog at the level of 8th thoracic vertebra.

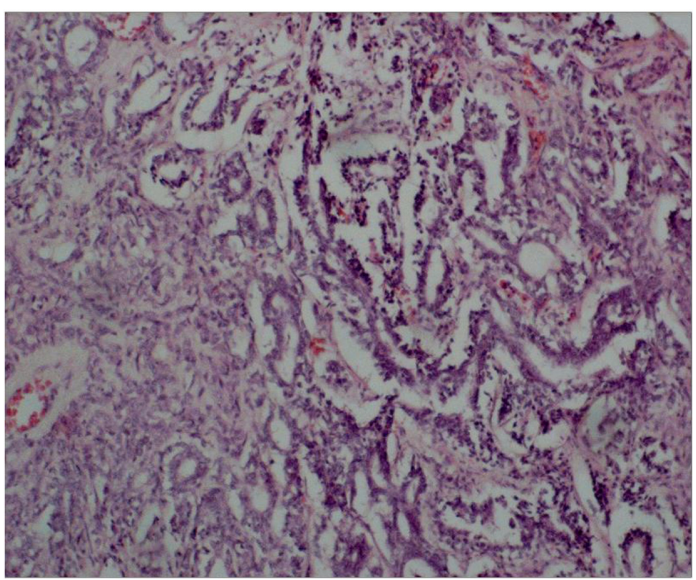

Fig. 4. Histopathologic preparation of intramuscular mass. Regular pattern of cuboidal and columnar epithelial cells in acinar space reveals mammary adenocarcinoma. H\&E Stain, $\times 200$.

In this case, there was a transformation of the muscle with lung metastasis. This is a rare metastasis and the reason is thought to be due to the incomplete removal of tumors in the previous resection. It have been the transition to the muscle through formation of vascular and suppression of the host immune system. To prevent this metastasis of tumor cells, accurate diagnosis of the tumor in the initial stage, and its completely surgical removal is required. Cytological differentiation between benign and malignant canine mammary tumors can be useful [1]. However, a definitive diagnosis of mammary tumors requires histopathologic confirmation [14].

Surgery remains the treatment of choice for dogs and cats with most types of mammary gland tumors, except for inoperable disease and organ metastases [12]. Early and complete resection and microscopic diagnostic examination are recommended. Delaying surgery may result in a larger tumor and a more difficult removal [6]. The extent of resection is 
determined by size, the degree of infiltration and the location of the tumor, the number of tumors, and the status of the regional lymph node. Lumpectomy should be restricted to small (less than $5 \mathrm{~mm}$ ), firm and non-fixed nodules. Simple mastectomy may be indicated if there is a single tumor in the thoracic gland, since no lymphatic connection exists with other glands [4, 15]. Regional mastectomy is considered in dogs with tumors that involve the abdominal and inguinal tumors or the second thoracic gland. In addition, there is chemotherapy, including doxorubicin, cyclophosphamide, cisplatin, and radiation therapy that is considered in a few dogs with inoperable tumors and in inflammatory carcinoma. Adjuvant ovariectomy had no real benefit to dogs with mammary cancer since most metastases lack hormone receptors [11]. In this case, although there was no recurrence of mammary gland cancer, the dog was euthanized due to metastasis and severe hypoglycemia as a paraneoplastic syndrome. It may have been caused by incomplete resection of primary tumor without accurate diagnosis one year ago.

Malignant MGT metastasize by hematogenous and lymphatic routes, and common metastatic sites include regional lymph nodes, lungs, adrenal gland, kidney and liver [2]. Therefore, in this case, Longissimus lumborum muscle metastasis from mammary gland adenocarcinoma is an unusual finding. Malignant cells can travel through the lymphatic system, which has its own channels throughout the body, and is regularly compared to the circulatory system [7]. When surgeons remove a tumor, they may also remove nearby portions of the lymph system, including the lymph nodes, as these are frequently the first sites of the cancers' metastasis. Once metastasis to the lymphatic system has occurred, the prognosis for cure drops significantly.

Malignant mammary tumours often metastasize into local lymphnodes and lungs, and less frequently into other organs. In this case, metastasizing adenocarcinoma was found in the lungs as well as within the tumour mass of the Longissimus lumborum muscle. This is a rare observation and could easily be overlooked or misinterpreted.

\section{References}

1. Allen SW, Prasse KW, Mahaffey EA. Cytologic differentiation of benign from malignant canine mammary tumors. Vet pathol 1986, 23, 649-655.
2. Fidler IJ, Brodey RS. A necropsy study of canine malignant mammary neoplasms. J Am Vet Med Assoc 1967, 151, 710-715.

3. Hamilton JM. Comparative aspects of mammary tumors. Adv Cancer Res 1974, 19, 1-45.

4. Hedlund CS. Surgery of the reproductive and genital systems. In: Fossum TW (ed.). Small Animal Surgery. 2nd ed. pp. 632-637, Mosby, St. Louis, 2002.

5. Kim NS, Choi OK, Jung IS, Choi IH. Application of Zplasty for skin defect by mastectomy in a dog. J Vet Clin 2002, 19, 440-442.

6. Misdorp W. Progestagens and mammary tumours in dogs and cats. Acta Endocrinol (Copenh) 1991, 125 (Suppl 1), 27-31.

7. Misdorp W, Hart AA. Canine mammary cancer. II. Therapy and causes of death. J Small Anim Pract 1979, 20, 395-404.

8. Morrison WB. Cancer in Dogs and Cats. 2nd ed. pp. 731744, Teton NewMedia, Jackson, 2002.

9. Priester WA, McKay FW. The occurrence of tumors in domestic animals. Natl Cancer Inst Monogr 1980, 54, 1210.

10. Robbins M. Reproductive oncology. In: Slatter D (ed.). Textbook of Small Animal Surgery. 3rd ed. pp. 2439-2443, Saunders, Philadelphia, 2003.

11. Rutteman GR, Withrow SJ, MacEwen EG. Tumors of the mammary gland. In: Withrow SJ, MacEwen EG (eds.). Small Animal Clinical Oncology. 3rd ed. pp. 461-463, Saunders, Philadelphia, 2001.

12. Sartin EA, Barnes $\mathbf{S}$, Toivio-Kinnucan $\mathbf{M}$, Wright JC, Wolfe LG. Heterogenic properties of clonal cell lines derived from canine mammary carcinomas and sensitivity to tamoxifen and doxorubicin. Anticancer Res 1993, 13, 229-236.

13. Schneider R, Dorn CR, Taylor DO. Factors influencing canine mammary cancer development and postsurgical survival. J Natl Cancer Inst 1969, 43, 1249-1261.

14. Sorenmo K. Canine mammary gland tumors. Vet Clin North Am Small Anim Pract 2003, 33, 573-596.

15. Stockhaus C, Kohn B, Rudolph R, Brunnberg L, Giger U. Correlation of haemostatic abnormalities with tumour stage and characteristics in dogs with mammary carcinoma. J Small Anim Pract 1999, 40, 326-331.

16. Susaneck SJ, Allen TA, Hoopes J, Withrow SJ, Macy DW. Inflammatory mammary carcinoma in the dog. J Am Anim hosp Assoc 1983, 19, 971-976. 